\title{
Cognitive Behavioral Based Treatment for Procrastination
}

\author{
Gabriel Talask* and Marcele Regine de Carvalho \\ Institute of Psychology, Federal University of Rio de Janeiro, Brazil.
}

Submission: December 05, 2017; Published: December 13, 2017

*Corresponding author: Gabriel Talask Moura, Institute of Psychology, Federal University of Rio de Janeiro, Brazil,

E-mail: gabrieltalask@gmail.com

\section{Introduction}

Procrastination is highly prevalent, being present in $20 \%$ of the general population [1-3]. When related to the academic domain, it can reach up to $70 \%$ [4]. In addition, procrastination affects different life-domains, compromising physical and mental health [5-7], finances [8,9] and individual performance and wellbeing [10-12]. Procrastination is more than a simply delay of a task or a decision to make; it also involves behavioral, cognitive and emotional aspects $[13,14]$. Understanding the complexity of its nature is fundamental in order to develop an effective intervention for its treatment. Although recent, academic studies are closer to a commonly shared theory. Therefore, the aim of this study is to present the mainly theories about the expression and nature of procrastination in order to review recent interventions for the treatment of procrastination.

\section{Understanding Procrastination}

Procrastination has been investigated from two perspectives: a) as an avoidant behavior that follows a specific task, culminating in the delay of this task; b) as a personality trait, being a chronic and diffuse pattern, in which delay and avoidance are typical responses in different situations [4]. While understanding procrastination as a personality trait, studies sought to locate it in the Big Five taxonomy [15]. Regarding this model, research suggests that procrastination is highly related with low level of Conscientiousness and moderate related with high level of Neuroticism [13,16-18]. All facets of Conscientiousness were inversely related to procrastination; nevertheless self-discipline appears as the greatest predictor, followed by organization [19]. As for Neuroticism, impulsivity and vulnerability are the most significant facets as predictors of procrastination, but also shows a relationship with the depression and self-awareness facets $[13,16,18]$.

\section{Predictors and Precursors of Procrastination}

Deficit in volition and failure of self-regulation represent some of the main predictors of procrastination. Procrastination appears as a gap between the implementation of an intention and the implementation of the action, causing the individual to procrastinate against his initial intention. Choosing to voluntarily delay an action, despite the individual's intention, is the fundamental point of self-regulatory failure $[18,20,21]$. procrastinatory behavior has also been identified as a coping strategy and as a way to regulating, in the short term, negative emotions that follows a certain task [22]. Some procrastinators experience specific ruminations about procrastination as an automatic thought about their own tendency to procrastinate (e.g. "Why I did not start this earlier?'). It is suggested that these ruminations not only follow procrastinatory behavior, they also precede task engagement. In that case, such cognitions would be a kind of worry. (e.g. People expect me to work and study more»). Therefore, specific cognitions linked to procrastination can lead to a broad spectrum of self-criticism and self-deprecating thoughts $[23,24]$. Procrastination is also evoked by certain situational aspects. Variables associated with procrastination are: attractiveness, difficulty and specificity of the task [18]. The more intrinsically unpleasant a task is, the more likely the individuals will be to postpone it. There is a natural tendency to avoid aversive stimuli, and the more aversive the stimulus, the greater the chances of evasive behavior. In addition, while procrastinating, individuals tend to distort their perception of the degree of importance, difficulty, pleasure, and stress of a task [22].

\section{Strategies for the Treatment of Procrastination}

Faced with this broad scenario that composes the expression of procrastination, strategies that involve stimulus control, emotional regulation, cognitive restructuring, and behavioral activation seem to have a degree of importance in managing this problem. Strategies related to stimuli control seems to be effective in managing procrastinatory behavior, since impulsiveness is a strong predictor of this behavior, and is also related to a greater susceptibility to engagement in activities involving immediate rewards. Such a strategy involves changes in the environment and situational factors, controlling the amount 
of stimuli that can contribute to the reduction of attention, as well as creating obstacles in the engagement of activities that promote immediate rewards [25]. The deficit in self-regulation is one of the mainly aspect of procrastination, which can be also a maladaptive strategy to deal with negative emotions [26]. Therefore, strategies involving acceptance and mindfulness have been shown to be effective in managing procrastination [27-29].

Given the influence of cognitive activity on behavior, cognitive restructuring is one of the main strategies and objectives of cognitive-behavioral psychotherapy [30,31]. Chronic procrastinators have certain thoughts related to procrastination which leads to negative emotional reactions, contributing to procrastinatory behavior $[14,24]$. Identifying and questioning these dysfunctional thoughts is fundamental in the management of this behavioral response. Procrastinators frequently do not initiate certain actions because they erroneously expect to create a prior motivation for such, or because they have a distorted perception about the difficulty and displeasure that a certain task will provide [22]. In this sense, behavioral activation presents itself as an important strategy in the management of behavior, since it consists of a strategy of engagement in activities that are often neglected in the individual's life, leading to a state of constant inactivity [32].

\section{Discussion and Conclusion}

Recent experimental researches [33-35] suggest that interventions based in Cognitive-Behavioral Therapy (CBT) show promise in the treatment of procrastination. Those studies showed a significant reduction in the scores on the scales used to measure procrastination, which were maintaining on the follow-up. However, some limitations need to be discussed. Those researches used different protocols, varying in strategies, interventions and assessments. In addition, there was also variation in the samples. A recent epidemiological study of procrastination [3] revealed that age, gender and education are relevant variables. Furthermore, only one trial used a randomized controlled trial. Although research has shown promising results in reducing procrastination, it is important that future research consider some factors for the consolidation of an evidence-based practice. It is necessary that studies sought to perform controlled and randomized clinical trials.

That kind of study is fundamental to determinate more specific outcomes, once it provides better control under the variables, such as psycho education, home practice and interaction with the professional. In addition, randomized sample allows considering co variables - such as age, gender and education - decreasing the risk of bias. Moreover, homogeneity among assessments is fundamental in order to discuss the outcomes. Procrastination is extremely prevalent and causes significant impairment in the lives of these people. Therefore, it is essential that there be evidence-based interventions for the treatment and management of this problem. However, for this to occur it is necessary that there be more studies that seek to understand the nature of procrastination, so experimental researches can converge in protocols. Although treatment studies are scarce and recent, interventions in cognitivebehavioral therapy have shown promise.

\section{References}

1. Ferrari JR, Doroszko E, Joseph N (2005) Exploring procrastination in corporate settings: Sex status and settings for arousal and avoidance types. IndividDiffer Res 3(2): 140-149.

2. Ferrari JR, Díaz Morales JF (2007) Procrastination: Different time orientations reflect different motives. J Res Pers 41(3): 707-714.

3. Steel P, Ferrari J (2013) Sex education and procrastination: an epidemiological study of procrastinators characteristics from a global sample. Eur J Pers 27(1): 51-58.

4. Schouwenburg HC (2004) Academic procrastination: Theoretical notions, measurement and research. In HC Schouwenburg, CH Lay, TA Pychyl, JR Ferrari (Eds.), Counseling the procrastinator in academic settings. American Psychological Association Washington, USA, pp. 3-17.

5. Tice DM, Baumeister RF (1997) Longitudinal study of procrastination performance stress and health: The costs and benefits of dawdling. Psychol Sci 8(6): 454-458.

6. Sirois FM, Melia Gordon ML, Pychyl TA (2003) “I'll look after my health, later" An investigation of procrastination and health. PersIndividDif 35(5): 1167-1184.

7. Sirois FM (2007) "I'll look after my health later" A replication and extension of the procrastination health model with community dwelling adults. PersIndividDif 43(1):15-26.

8. O Donoghue R, Rabin M (1998) Procrastination in preparing for retirement. In HJ Aaron (Eds.), Behavioral dimensions of retirement economics. Brooking Institution Press, New York, USA, pp. 125-156.

9. Byrne A, Blake D, Cairns A, Dowd K (2006) There's no time like the present: The cost of delaying retirement saving. Financial Services Review 15(3): 213-231.

10. Ferrari JR, Barnes KL, Steel P (2009) Life regrets by avoidant and arousal procrastinators Why put off today what you will regret tomorrow? J Individ Differ 30(3): 163-168.

11. Pychyl TA (2013) Solving the procrastination puzzle: A concise guide to strategies for change. Penguin Group, New York, USA.

12. Tibbett TP, Ferrari JR (2015) The portrait of the procrastinator: Risk factors and results of an indecisive personality. PersIndividDif 82: 175184.

13. Schouwenburg HC, Lay CH (1995) Trait procrastination and the Big Five factors of personality. PersIndividDif 18(4): 481-490.

14. Stainton M, Lay CH, Flett GL (2000) Trait procrastinators and behavior/ trait-specific cognitions. J SocBehavPers 15(5): 297-312.

15. Costa PT, McCrae RR (1992) The five-factor model of personality and its relevance to personality disorders. J PersDisord 6(4): 343-359.

16. Watson DC (2001) Procrastination and the five-factor model: A facet level analysis. PersIndividDif 30(1): 149-158.

17. Van Eerde W (2003) A meta-analytically derived nomological network of procrastination. PersIndividDif 35(6): 1401-1418.

18. Steel $P$ (2007) The nature of procrastination: A meta-analytic and theoretical review of quintessential self-regulatory failure. Psychol Bull 133(1): 65-94.

19. Díaz Morales J, Cohen J, Ferrari J (2008) An integrated view of personality styles related to avoidant procrastination. PersIndividDif 45(6): 554-558. 
20. Pychyl TA, Flett GL (2012) Procrastination and Self Regulatory Failure: An Introduction to the Special Issue. J RationEmotCognBehavTher 30(4): 203-212

21. Sirois F, Pychyl T (2013) Procrastination and the priority of short term mood regulation: Consequences for future self. SocPersonalPsychol Compass 7(2): 115-127.

22. Pychyl TA, Lee JM, Thibodeau R, Blunt A (2000) Five days of emotion: An experience sampling study of undergraduate student procrastination. SocBehavPers 15(5): 239-254.

23. Lay CH, Silverman S (1996) Trait procrastination anxiety and dilatory behavior. PersIndividDif 21(1): 61-67.

24. Flett GL, Stainton M, Hewitt PL, Sherry SB, Lay C (2012) Procrastination automatic thoughts as a personality construct: an analysis of the procrastinator cognitions inventory. J RationEmotCognBehavTher 30(4): 223-236

25. Rozental A, Carlbring P (2014) Understanding and Treating Procrastination: A Review of a Common Self-Regulatory Failure. Psychology 5(13): 1488-1502.

26. Basco MR (2010) The procrastinators guide to getting things done. New York, USA.

27. Glick DM, Orsillo SM (2015) An Investigation of the Efficacy of Acceptance-Based Behavioral Therapy for Academic Procrastination. J ExpPsycholGen 144(2): 400-409.
28. Scent CL, Boes SR (2014) Acceptance and Commitment Training: A Brief Intervention to Reduce Procrastination Among College Students. J College Stud Psychother 28(2): 144-156.

29. Flett AL, Haghbin M, Pychyl TA (2016) Procrastination and depression from a cognitive perspective: An exploration of the associations among procrastinator automatic thoughts, rumination, and mindfulness. J RationEmotCognBehavTher 34(3): 169-186.

30. Knapp P et al. (2004) Terapia cognitivo-comportamental na prática psiquiátrica. Porto Alegre Artmed.

31. Hofmann SG (2014) Introdução à terapia cognitivo-comportamental contemporânea. Porto Alegre Artmed.

32. Beck J (2013) Terapia cognitivo comportamental. Porto Alegre Artmed.

33. Karas D, Spada MM (2009) Brief cognitive-behavioral coaching for procrastination: A case series. Coaching An International Journal of Theory 2(1):44-53.

34. Ozer BU, Demir A, Ferrari JR (2013) Reducing academic procrastination through a group treatment program A pilot study. J Ration Emot Cogn Behav Ther 31(3): 127-135.

35. Rozental A, Forsell E, Svensson A, Andersson G, Carlbring P (2015) Internet based cognitive behavior therapy for procrastination $\mathrm{A}$ randomized controlled trial. J ConsultClinPsycho 83(4): 808-824.

\section{Your next submission with Juniper Publishers will reach you the below assets}

- Quality Editorial service

- Swift Peer Review

- Reprints availability

- E-prints Service

- Manuscript Podcast for convenient understanding

- Global attainment for your research

- Manuscript accessibility in different formats

( Pdf, E-pub, Full Text, Audio)

- Unceasing customer service

Track the below URL for one-step submission https://juniperpublishers.com/online-submission.php 